\title{
REGENERAÇÃO NATURAL DE FLORESTA ESTACIONAL SEMIDECIDUAL EM DIFERENTES ESTÁGIOS SUCESSIONAIS (ZONA DA MATA, MG, BRASIL)
}

\author{
Sustanis Horn Kunz ${ }^{1}$; Sebastião Venâncio Martins ${ }^{2}$ \\ ${ }^{1}$ Bióloga, Dra ${ }^{\mathrm{a}}$, Depto. de Ciências Florestais e da Madeira, UFES, Alegre, ES, Brasil - sustanis.kunz@ufes.br \\ ${ }^{2}$ Eng. Florestal, Dr., Depto. de Engenharia Florestal, UFV, Viçosa, MG, Brasil - venancio@ufv.br \\ Recebido para publicação: 01/02/2013 - Aceito para publicação: 25/10/2013
}

\begin{abstract}
Resumo
O objetivo deste estudo foi avaliar a estrutura e a composição florística da regeneração natural de trechos de Floresta Estacional Semidecidual em diferentes estágios sucessionais. Para a estrutura fitossociológica, foram demarcadas sistematicamente 10 parcelas de 5 x $10 \mathrm{~m}$ em trecho de floresta em estágio médio de regeneração (FEM), floresta em estágio avançado de regeneração (FEA) e pastagem abandonada (PAS), sendo amostrados os indivíduos com altura $\geq 50 \mathrm{~cm}$ e diâmetro à altura do solo $\leq 5 \mathrm{~cm}$. Foi realizada análise de correspondência retificada e análise de espécies indicadoras de cada trecho. Na estrutura da regeneração natural da FEM e da pastagem, houve destaque de apenas uma espécie (Psychotria sessilis e Vernonia polyanthes, respectivamente) quanto ao Valor de Importância (VI), devido principalmente à alta densidade. Já na FEA não foi observada forte dominância por uma espécie. As três áreas apresentaram-se distintas quanto à abundância e composição de espécies, sugerindo que a matriz florestal é composta por um mosaico sucessional. Além disso, cada trecho ainda mantém espécies indicadoras características, de acordo com o estágio sucessional em que se encontram, razão pela qual ações de manejo e enriquecimento poderiam ser adotadas para acelerar o processo de sucessão.

Palavras-chave: Composição florística; conservação; estrutura; sucessão secundária.
\end{abstract}

\begin{abstract}
Natural regeneration of seasonal semideciduous forest in different successional stages (Zona da Mata, MG, Brazil). The objective of this study was to evaluate the floristic structure and composition of the natural regeneration of stretches from Seasonal Semideciduous Forest in different successional stages. Ten plots, 5 x 10 meters in size, were systematically delimited for phytosociological structure in patches of forest which were in intermediate regeneration stage (FEM), advanced regeneration stage (FEA) and abandoned pasture (PAS).We sampled the individuals with $\geq 50 \mathrm{~cm}$ in height and diameter at ground height $\leq 5 \mathrm{~cm}$. We performed Detrended Correspondence Analysis and analysis of indicative species of each forest section. In the natural regeneration structure of FEM and pasture it was featured only one species (Psychotria sessilis and Vernonia polyanthes, respectively) in the Importance Value (IV), mainly due to high density. In relation to the FEA there was no strong dominance by one species. The three sections showed distinct species abundance and composition, which suggests that the forest matrix comprises a successional mosaic. Furthermore, each stretch still maintains indicative characteristic species, according to the successional stage they are in. Because of this, management and enrichment actions could be performed to accelerate the succession process.

Keywords: Floristic composition; conservation; structure; secondary succession.
\end{abstract}

\section{INTRODUÇÃO}

A maior parte das áreas de Floresta Atlântica brasileira é constituída por formações secundárias em diferentes estágios sucessionais, que se encontram extremamente fragmentadas em função do uso e ocupação do solo desde o período de colonização do país (MORELLATO; HADDAD, 2000). O uso insustentável dos recursos naturais, associado ao desmatamento, há décadas vem produzindo extensas áreas degradadas, inclusive em fragmentos florestais sujeitos a pressões antrópicas. Associados ao grau de 
degradação da área, alguns fatores, como limitação da dispersão, predação de sementes e plântulas e competição por gramíneas, podem diminuir ou impedir a regeneração natural de florestas tropicais em locais sujeitos a fortes perturbações ou que foram desmatados e depois abandonados (NOTMAN; GORCHOV, 2001; GASPARINO et al., 2006; MARTINEZ-GARZA et al., 2011). Uma vez abandonadas, o processo de regeneração natural pode ser ausente, devido ao elevado nível de degradação (HOOPER et al., 2005), ou muito lento, podendo resultar em um ecossistema distinto do original (CAVELIER et al., 1998). No outro extremo, se áreas desmatadas foram usadas por poucos anos (aproximadamente 2 anos) e estão inseridas em uma matriz florestal, é possível que em poucas décadas (aproximadamente 4) a estrutura fitofisionômica dessas áreas seja semelhante à das florestas do entorno, apenas com o processo de regeneração natural (AIDE et al., 2000).

Existem poucos estudos que abordam a análise da regeneração natural em diferentes estágios sucessionais de uma mesma matriz florestal. Dentre eles, destacam-se os estudos de Pereira et al. (2001), na Caatinga, o de Schorn e Galvão (2006), realizado em Floresta Ombrófila Densa em Santa Catarina, e o de Rayol et al. (2008), em Floresta Ombrófila secundária no Pará. No trabalho de Aide et al. (2000), foi estudada a regeneração natural de uma cronossequência de pastagens abandonadas em Porto Rico e foi observado que não houve diferença significativa para os parâmetros densidade e riqueza de espécies entre florestas secundárias de 40 anos de idade e floresta madura (80 anos). Contudo, os autores encontraram diferenças significativas na composição florística entre tais estágios sucessionais. Em Floresta Estacional Semidecidual, Ribas et al. (2003) encontraram baixa similaridade florística da regeneração natural, ao analisarem a composição florística de dois trechos com diferentes estágios de sucessão secundária (15 e 30 anos). Silva Júnior et al. (2004) estudaram o componente da regeneração natural em dois trechos de floresta estacional semidecidual em Minas Gerais e observaram diferenças na composição florística e abundância das espécies arbustivo-arbóreas entre os trechos de floresta madura e floresta inicial.

Nesse contexto, percebe-se grande lacuna no que diz respeito à caracterização estrutural da regeneração de áreas com diferentes estágios sucessionais de um mesmo fragmento florestal, cujo conhecimento é fundamental para avaliar o estado de conservação de áreas sem perturbações antrópicas e definir estratégias que possam acelerar o curso da sucessão de áreas antropizadas.

A Estação de Pesquisa, Treinamento e Educação Ambiental Mata do Paraíso, localizada no município de Viçosa, MG, apresenta uma paisagem composta por áreas de floresta secundária média e madura e áreas de pastagem abandonada de capim-gordura (Melinis minutiflora $\mathrm{P}$. Beauv.). Considerando que esses três trechos estão interligados em um mosaico na mesma matriz ambiental, foram formuladas as seguintes perguntas: (1) Está havendo direcionamento florístico/estrutural da pastagem para a floresta secundária média e desta para a floresta secundária tardia? (2) As espécies indicadoras já são de categorias sucessionais subsequentes a cada trecho sucessional? Para responder essas perguntas, o objetivo deste estudo foi avaliar a regeneração natural de dois trechos de floresta em diferentes estágios sucessionais (floresta madura e floresta secundária) e de pastagem abandonada, por meio da estrutura fitossociológica, composição florística e análise de espécies indicadoras de cada ambiente.

\section{MATERIAL E MÉTODOS}

O estudo foi realizado na Estação de Pesquisa, Treinamento e Educação Ambiental Mata do Paraíso (EPTEA), localizada nas coordenadas $20^{\circ} 48^{\prime} 07^{\prime \prime} \mathrm{S}$ e $42^{\circ} 51^{\prime} 31^{\prime \prime} \mathrm{W}$, no município de Viçosa, Minas Gerais. A EPTEA possui 195 ha de área e altitude variando de 690 a $800 \mathrm{~m}$ (BRAZ et al., 2002). O clima na região é do tipo Cwb (KÖPPEN, 1948), com temperatura média anual de $21,8{ }^{\circ} \mathrm{C}$ e precipitação pluviométrica média anual de 1314,2 mm (CASTRO et al., 1983). A vegetação da reserva é composta por trechos de Floresta Estacional Semidecidual, segundo Instituto Brasileiro de Geografia e Estatística (IBGE) (2012), e o relevo é do tipo ondulado a fortemente ondulado (CORRÊA, 1984). Os solos predominantes são o Latossolo Vermelho-Amarelo e o Argissolo Vermelho-Amarelo (SANTOS et al., 2013).

Os trechos de floresta selecionados para a amostragem da regeneração natural apresentam diferentes históricos de perturbação e regeneração. O trecho denominado neste estudo de floresta em estágio médio de regeneração (FEM), segundo a resolução CONAMA n 392, de 2007 (BRASIL, 2013), foi totalmente suprimido para pecuária. Em 1963, a área foi abandonada, estando há 47 anos (até a realização do presente estudo) em processo de regeneração natural. O outro trecho, denominado floresta 
em estágio avançado de regeneração (FEA), segundo a mesma resolução do CONAMA, sofreu somente exploração seletiva de madeira e está livre de distúrbios antrópicos nas últimas quatro décadas (SILVA JÚNIOR et al., 2004). Um terceiro trecho é caracterizado por pastagem abandonada (PAS) de capimgordura (Melinis minutiflora P. Beauv) e configura-se como uma grande clareira antrópica dentro da matriz florestal da EPTA.

Em cada trecho, foram demarcadas sistematicamente dez parcelas de 5 x 10 m, com distância de $10 \mathrm{~m}$ entre parcelas subsequentes. Foram incluídos todos os indivíduos arbustivo-arbóreos com altura $\geq 50 \mathrm{~cm}$ e diâmetro à altura do solo (DAS) $\leq 5 \mathrm{~cm}$, segundo preconizado por Durigan et al. (2002). Os indivíduos foram identificados por meio de comparação com o material do Herbário VIC da Universidade Federal de Viçosa, consulta à literatura especializada e especialistas, sempre que necessário. Os nomes científicos e respectivos autores foram atualizados de acordo com o Missouri Botanical Garden (2012), tendo sido adotado o sistema Angiosperm Philogeny Group III (APG, 2009).

Os parâmetros fitossociológicos da regeneração natural (densidade, frequência, dominância e valor de importância), a diversidade de Shannon-Wiener ( $\left.\mathrm{H}^{\prime}\right)$ e a Equabilidade de Pielou foram calculados de acordo com Mueller-Dombois e Ellenberg (1974), por meio do programa FITOPAC 2.01 (SHEPHERD, 2009). As espécies foram classificadas nas categorias sucessionais pioneiras, secundárias iniciais e secundárias tardias, de acordo com os trabalhos realizados na região (SILVA JÚNIOR et al., 2004; ARAÚJO et al., 2006; HIGUCHI et al., 2006; MARTINS et al., 2008). Considerando ainda o índice de Shannon, aplicou-se o teste $t$ de Hutcheson (1970), a fim de comprovar a semelhança ou não da diversidade $\left(\mathrm{H}^{\prime}\right)$ entre os fragmentos. Essa análise foi realizada pelo programa PAST versão 2.16 (HAMMER et al., 2012).

Foi elaborada uma matriz de abundância das espécies de cada área, para determinação das semelhanças de estrutura, por meio da análise de correspondência retificada (Detrendend Correspondence Analyses - DCA) (HILL; GAUCH, 1980). Para se testar a diferença na composição de espécies entre os estágios sucessionais, aplicou-se a análise de similaridade bifatorial (ANOSIM). Todas as análises foram realizadas no programa R (R DEVELOPMENT CORE TEAM, 2009). As espécies indicadoras de cada estágio sucessional foram obtidas com a análise de espécies indicadoras (ISA) (DUFRÊNE; LEGENDRE, 1997), por meio do programa PC-ORD 5 (MCCUNE; MEFFORD, 2006). Em função da grande heterogeneidade para os grupos indicada pelo resultado do ISA, foi estabelecido um limite mínimo de 70\% de valor indicador, aliado ao grau de significância, para identificação das espécies indicadoras de cada grupo.

\section{RESULTADOS E DISCUSSÃO}

Considerando os três trechos estudados, foram amostradas 114 espécies, distribuídas em 77 gêneros e 37 famílias. Os parâmetros gerais do estrato de regeneração natural de cada trecho estão apresentados na tabela 1 .

Tabela 1. Parâmetros gerais da regeneração natural dos trechos de floresta em estágio avançado de regeneração (FEA), floresta em estágio médio (FEM) e pastagem (PAS), amostrados na EPTEA Mata do Paraíso, Viçosa, MG.

Table 1. General parameters of the natural regeneration of stretches of middle state forest, initial forest and pasture, sampled at the EPTEA Mata do Paraíso, Viçosa, MG.

\begin{tabular}{lccc}
\hline Parâmetros & FEA & FEM & PAS \\
\hline Número de indivíduos & 1288 & 1386 & 105 \\
Número de espécies & 67 & 60 & 21 \\
Número de famílias & 25 & 27 & 11 \\
Densidade (ind./m ${ }^{2}$ ) & 2576 & 2772 & 210 \\
Diversidade (H') & 3,06 & 1,63 & 2,17 \\
Equabilidade (J') & 0,72 & 0,40 & 0,71 \\
\hline
\end{tabular}

O trecho de floresta em estágio avançado de regeneração possui maior riqueza florística em relação às outras áreas (floresta em estágio médio e pastagem), mas não foi observada diferença significativa quanto à diversidade ( $\mathrm{p}>0,001)$, quando comparada aos pares com a floresta em estágio 
médio e a pastagem. A FEM foi o trecho com menor diversidade florística $\left(\mathrm{H}^{\prime}=1,63\right)$, havendo diferença significativa $(\mathrm{p}<0,001)$ apenas em relação à pastagem. A baixa equabilidade encontrada para a FEM $\left(J^{\prime}=0,40\right)$ provavelmente é devido à alta abundância de Psychotria sessilis Vell. (Tabela 2), perfazendo $67 \%$ do número de indivíduos amostrados para a comunidade. Segundo Gomide et al. (2006), essa dominância ecológica da espécie contribui para a baixa diversidade local. Na pastagem, Vernonia polyanthes foi a espécie mais abundante (Tabela 2), representando $45 \%$ dos indivíduos amostrados, enquanto os demais estão distribuídos de maneira mais uniforme entre as espécies, garantindo uma equabilidade semelhante à FEA. Apesar disso, a baixa diversidade na PAS possivelmente é reflexo do número de espécies encontrado, pois os valores de diversidade também estão relacionados à quantidade de espécies amostradas (MAGURRAN, 2013). Estudos mostram que a diversidade de espécies aumenta de acordo com a idade da comunidade em florestas tropicais (SALDARRIAGA et al., 1988; ANDEL, 2001), sendo que o número de espécies pioneiras diminui à medida que a sucessão avança, enquanto as espécies tardias se estabelecem lentamente na comunidade (SALDARRIAGA et al., 1988). Esses autores observaram que, entre 30 e 40 anos, espécies secundárias iniciais podem ser substituídas por grupos de espécies de crescimento rápido, mas persistentes, tornando-se dominantes no ambiente tanto em densidade quanto em área basal e ainda podem permanecer assim na fase de floresta madura.

Psychotria sessilis, que exibe alta dominância na floresta em estágio médio de regeneração do presente estudo (Tabela 2), parece seguir o mesmo padrão observado por Saldarriaga et al. (1988). Essa espécie, classificada como secundária inicial, também se destacou em VI num sub-bosque de povoamento de eucalipto em Viçosa, MG (SOUZA et al., 2007). Em estudo de banco de sementes desse mesmo trecho, $P$. sessilis foi uma das espécies arbóreas que apresentaram maior número de indivíduos germinados, o que lhe confere alta produção de sementes aliada à facilidade de germinação (KUNZ, 2011), sugerindo que possivelmente permanecerá na comunidade por longo período de tempo.

No trecho de pastagem, foi observada baixa abundância, e as espécies que o compõem são principalmente representadas por arbustos ruderais, cujas espécies não foram encontradas nos trechos de florestas em estágio avançado e médio de regeneração. O tempo em que está abandonada (cerca de 40 anos) e o fato de estar inserida em uma matriz florestal poderiam propiciar à pastagem a ocorrência de maior número de indivíduos, preferencialmente lenhosos de porte arbóreo, mas possivelmente a espécie Melinis minutiflora (capim-gordura) continua exercendo forte pressão sobre a sucessão da comunidade lenhosa na área. A densa camada epígea das gramíneas agressivas pode impedir a chegada de propágulos no solo (MARTINS; RIBEIRO, 2002), motivo pelo qual a proximidade de fontes de propágulos provavelmente não está sendo suficiente para promover o estabelecimento e desenvolvimento de espécies arbustivo-arbóreas nativas na área dominada por gramíneas. Esse impedimento no avanço da sucessão é exemplo típico do modelo de inibição proposto por Connell e Slatyer (1977), segundo o qual uma espécie é capaz de impedir o avanço da sucessão, deixando a comunidade estagnada quanto à recuperação da estrutura da comunidade arbustivo-arbórea, da riqueza e da diversidade florística.

As dez espécies de maior Valor de Importância (VI) de cada trecho não estão representadas nos demais quando se considera esse índice (Tabela 2).

No trecho de pastagem e de floresta em estágio médio de regeneração, apenas uma espécie destacou-se em VI (Tabela 2), reflexo principalmente da alta abundância de Psychotria sessilis em FEM e de Vernonia polyanthes Less. na pastagem. Provavelmente, a abundância de indivíduos propiciou os maiores valores de dominância relativa a essas espécies (64,67 e 58,52\%, respectivamente). Para o trecho de floresta em estágio avançado, esse padrão não acontece, pois não houve destaque de apenas uma espécie. Coffea arabica L., que se destacou nesse trecho em VI, é representada por indivíduos finos e, por isso, mesmo com alta abundância (260 indivíduos), obteve valores menores de dominância, quando comparada com Picramnia regneli Engl. e Euterpe edulis Mart. (Tabela 2). Apesar disso, em algumas parcelas percebeu-se que a alta dominância de $C$. arabica está inibindo o desenvolvimento de espécies arbóreas, cujo fato é preocupante em termos de conservação, já que essas espécies, que ocuparão o dossel florestal futuramente, não estão conseguindo se desenvolver em alguns locais.

Estudos anteriores realizados no mesmo fragmento florestal demonstraram que $C$. arabica também apresentou maior densidade (SILVA JÚNIOR et al., 2004; PINTO et al., 2007; MARTINS et al., 2008), bem como em outros trechos de Floresta Estacional Semidecidual no estado de São Paulo (MARTINS; RODRIGUES, 2002; MARTINS et al., 2004). A alta abundância de $C$. arabica no trecho de FEA é reflexo da proximidade do fragmento florestal com áreas de plantios de café, cuja atividade era intensa na região há algumas décadas. A facilidade de dispersão de sementes, por ser zoocórica, aliada à adaptabilidade ao sub-bosque (DIAS et al., 2005) ou em clareiras (ARMELIN; MANTOVANI, 2001), faz com que seja destaque até em florestas maduras. 
Tabela 2. Parâmetros fitossociológicos para as dez espécies de maior Valor de Importância amostradas nos diferentes trechos em regeneração analisados na EPTEA Mata do Paraíso, Viçosa, MG.

Table 2. Phytosociological parameters for the ten species of higher Importance Value sampled from different regeneration stretches analyzed in the EPTEA Mata do Paraíso, Viçosa, MG.

\begin{tabular}{|c|c|c|c|c|c|c|c|c|}
\hline Espécies & NI & DA & DR & FA & FR & DoA & DoR & VI $(\%)$ \\
\hline \multicolumn{9}{|c|}{ Trecho de floresta em estágio avançado de regeneração } \\
\hline Coffea arabica $\mathrm{L}$. & 260 & 5200 & 20,19 & 80 & 3,42 & 0,64 & 10,96 & 11,52 \\
\hline Picramnia regneli Engl. & 125 & 2500 & 9,7 & 100 & 4,27 & 0,70 & 12,05 & 8,68 \\
\hline Euterpe edulis Mart. & 46 & 920 & 3,57 & 90 & 3,85 & 0,78 & 13,27 & 6,90 \\
\hline Trichilia lepidota Mart. & 62 & 1240 & 4,81 & 70 & 2,99 & 0,58 & 9,94 & 5,92 \\
\hline Guarea macrophyla Vahl & 77 & 1540 & 5,98 & 100 & 4,27 & 0,34 & 5,85 & 5,37 \\
\hline $\begin{array}{l}\text { Sorocea bonplandii (Baill.) } \\
\text { W.C. Burger, Lanj. \& Wess. }\end{array}$ & 62 & 1240 & 4,81 & 100 & 4,27 & 0,38 & 6,48 & 5,19 \\
\hline Guapira opposita (Vell.) Reitz & 68 & 1360 & 5,28 & 100 & 4,27 & 0,3 & 5,18 & 4,91 \\
\hline $\begin{array}{l}\text { Psychotria longepedunculata } \\
\text { Gardiner }\end{array}$ & 77 & 1540 & 5,98 & 70 & 2,99 & 0,24 & 4,14 & 4,37 \\
\hline Mollinedia widgrenii A. DC. & 49 & 980 & 3,80 & 90 & 3,85 & 0,26 & 4,42 & 4,03 \\
\hline Nectandra reticulata $\mathrm{Mez}$. & 66 & 1320 & 5,12 & 80 & 3,42 & 0,17 & 2,85 & 3,80 \\
\hline \multicolumn{9}{|c|}{ Trecho de floresta em estágio médio de regeneração natural } \\
\hline Psychotria sessilis Vell. & 926 & 18520 & 66,81 & 100 & 6,33 & 3,81 & 64,67 & 45,94 \\
\hline Trichilia lepidota Mart. & 86 & 1720 & 6,20 & 90 & 5,70 & 0,49 & 8,39 & 6,76 \\
\hline Siparuna guianensis Aubl. & 93 & 1860 & 6,71 & 90 & 5,70 & 0,38 & 6,48 & 6,29 \\
\hline $\begin{array}{l}\text { Erythroxylum pelleterianum A. } \\
\text { St.-Hil. }\end{array}$ & 32 & 640 & 2,31 & 60 & 3,80 & 0,16 & 2,75 & 2,95 \\
\hline $\begin{array}{l}\text { Anadenanthera macrocarpa } \\
\text { (Benth.) Brenan }\end{array}$ & 43 & 860 & 3,10 & 50 & 3,16 & 0,12 & 2,00 & 2,75 \\
\hline $\begin{array}{l}\text { Dalbergia nigra (Vell.) Allemao } \\
\text { ex Benth. }\end{array}$ & 19 & 380 & 1,37 & 60 & 3,80 & 0,12 & 2,10 & 2,42 \\
\hline $\begin{array}{l}\text { Nectandra oppositifolia Nees \& } \\
\text { Mart. }\end{array}$ & 18 & 360 & 1,30 & 80 & 5,06 & 0,04 & 0,66 & 2,34 \\
\hline Croton sp. & 11 & 220 & 0,79 & 60 & 3,80 & 0,05 & 0,87 & 1,82 \\
\hline Xylopia sericea A. St.-Hil. & 7 & 140 & 0,51 & 60 & 3,80 & 0,04 & 0,69 & 1,66 \\
\hline Leandra niangaeformis Cogn. & 13 & 260 & 0,94 & 60 & 3,80 & 0,01 & 0,09 & 1,61 \\
\hline \multicolumn{9}{|c|}{ Trecho de pastagem } \\
\hline Vernonia polyanthes Less. & 47 & 940 & 44,76 & 90 & 21,95 & 0,58 & 58,52 & 41,74 \\
\hline Solanum erianthum D. Don & 7 & 140 & 6,67 & 50 & 12,20 & 0,08 & 8,24 & 9,03 \\
\hline Vernonia westiniana Less. & 4 & 80 & 3,81 & 20 & 4,88 & 0,11 & 11,57 & 6,75 \\
\hline Ageratum conizoydes $\mathrm{L}$. & 11 & 220 & 10,48 & 20 & 4,88 & 0,03 & 3,20 & 6,19 \\
\hline Trema micrantha (L.) Blume & 4 & 80 & 3,81 & 30 & 7,32 & 0,02 & 1,75 & 4,29 \\
\hline Baccharis dracunculifolia DC. & 4 & 80 & 3,81 & 30 & 7,32 & 0,01 & 1,45 & 4,19 \\
\hline $\begin{array}{l}\text { Senna obtusifolia (L.) H.S. Irwin } \\
\text { \& Barneby }\end{array}$ & 3 & 60 & 2,86 & 20 & 4,88 & 0,01 & 1,37 & 3,03 \\
\hline Lantana camara $\mathrm{L}$. & 3 & 60 & 2,86 & 20 & 4,88 & 0,01 & 1,23 & 2,99 \\
\hline Schinus terebenthifolius Raddi & 5 & 100 & 4,76 & 10 & 2,44 & 0,01 & 1,41 & 2,87 \\
\hline Psidium guajava $\mathrm{L}$. & 3 & 60 & 2,86 & 10 & 2,44 & 0,02 & 1,96 & 2,42 \\
\hline
\end{tabular}

NI: número de indivíduos; DA: densidade absoluta; DR: densidade relativa (\%); FA: frequência absoluta; FR: frequência relativa (\%); DoA: dominância absoluta; DoR: dominância relativa (\%); VI (\%): valor de importância relativa.

No trecho de floresta em estágio avançado, as espécies de maior VI são classificadas como secundárias tardias, com exceção de Guapira opposita (Vell.) Reitz, que é secundária inicial, e $C$. arabica, sem caracterização (Tabela 2). Esse padrão condiz com o estágio sucessional desse trecho, em que as condições ambientais podem estar propiciando o estabelecimento de espécies mais exigentes. E. edulis, Guarea macrophyla Vahl e G. opposita estiveram entre as mais importantes na comunidade arbustivo-arbórea adulta desse trecho em estudo realizado por Pinto et al. (2007). Já C. arabica foi representada por apenas três indivíduos (DAP $\geq 4,8 \mathrm{~cm}$ ), provavelmente pelo fato de que essa espécie não atinge diâmetros superiores, permanecendo apenas no sub-bosque florestal. 
As espécies de maior VI na floresta em estágio médio de regeneração (Tabela 2) compreendem indivíduos regenerantes de arvoretas e árvores classificadas como secundárias iniciais e secundárias tardias (Tabela 3), demonstrando o potencial da comunidade para o avanço na sucessão. No componente da regeneração natural, ainda estão presentes espécies que retratam a condição jovem da floresta (GANDOLFI et al., 1995), como P. sessilis, E. pelleterianum e A. macrocarpa, classificadas como secundárias iniciais (HIGUCHI et al., 2006), assim como já estão estabelecidas espécies tardias de sucessão, como T. lepidota e S. guianensis (ARAÚJO et al., 2006; MARTINS et al., 2008), que se destacam na comunidade pela alta abundância e frequência nas unidades amostrais.

Tabela 3. Análise de espécies indicadoras e informações ecológicas das espécies e respectivas famílias amostradas nos trechos de floresta madura, floresta estágio médio de sucessão e pastagem, EPTEA Mata do Paraíso, Viçosa, MG.

Table 3. Indicator species analysis and ecological information of the sampled species and their families at the mature forest, initial forest and pasture sites at EPTEA Mata do Paraíso, Viçosa, MG.

\begin{tabular}{|c|c|c|c|c|c|c|c|}
\hline Família/Espécie & FEA & FEM & PAS & GS & GI & IV & $\mathbf{p}$ \\
\hline \multicolumn{8}{|l|}{ ACANTHACEAE } \\
\hline Geissomeria schottiana Nees & $\mathrm{x}$ & & & St & FM & 60 & 0,0006 \\
\hline \multicolumn{8}{|l|}{ ANACARDIACEAE } \\
\hline Schinus terebinthifolia Raddi & & & $\mathrm{x}$ & $\mathrm{P}$ & PAS & 10 & 1,0000 \\
\hline \multicolumn{8}{|l|}{ ANNONACEAE } \\
\hline Annona cacans Warm. & $\mathrm{x}$ & & & $\mathrm{Si}$ & FM & 10 & 1,0000 \\
\hline Guatteria nigrescens Mart. & $\mathrm{x}$ & & & St & FM & 30 & 0,0834 \\
\hline Rollinia sericea (R.E. Fr.) R.E. Fr. & $\mathrm{x}$ & & & $\mathrm{Si}$ & FM & 10 & 1,0000 \\
\hline Xylopia sericea A. St.-Hil. & & $\mathrm{x}$ & $\mathrm{x}$ & $\mathrm{P}$ & FI & 52,5 & 0,0061 \\
\hline \multicolumn{8}{|l|}{ ARALIACEAE } \\
\hline Schefflera morototoni (Aubl.) Maguire, Steyerm. & & $\mathrm{x}$ & & $\mathrm{P}$ & FI & 20 & 0,3030 \\
\hline \multicolumn{8}{|l|}{ ARECACEAE } \\
\hline \multirow{2}{*}{\multicolumn{8}{|c|}{ ASTERACEAE }} \\
\hline & & & & & & & \\
\hline Ageratum conyzoides $\mathrm{L}$. & & & $\mathrm{x}$ & $\mathrm{P}$ & PAS & 20 & 0,3089 \\
\hline Baccharis dracunculifolia DC. & & & $\mathrm{x}$ & $\mathrm{P}$ & PAS & 30 & 0,0847 \\
\hline Vernonia polyanthes Less. & & & $\mathrm{x}$ & $\mathrm{P}$ & PAS & 90 & $0,0001^{*}$ \\
\hline \multirow{2}{*}{\multicolumn{8}{|c|}{ BIGNONIACEAE }} \\
\hline & & & & & & & \\
\hline Jacaranda micrantha Cham. & & $\mathrm{x}$ & & $\mathrm{Si}$ & FI & 10 & 1,0000 \\
\hline \multicolumn{8}{|l|}{ BURSERACEAE } \\
\hline \multicolumn{8}{|l|}{ CANNABACEAE } \\
\hline Celtis iguanaea (Jacq.) Sarg. & $\mathrm{x}$ & $\mathrm{x}$ & & $\mathrm{P}$ & FM & 13,3 & 0,7530 \\
\hline Trema micrantha (L.) Blume & & & $\mathrm{x}$ & $\mathrm{P}$ & PAS & 30 & 0,0881 \\
\hline \multicolumn{8}{|l|}{ CARDIOPTERIDACEAE } \\
\hline $\begin{array}{l}\text { Citronella paniculata (Mart.) R.A. Howard } \\
\text { CLUSIACEAE }\end{array}$ & $\mathrm{x}$ & & & St & FM & 50 & 0,0043 \\
\hline Garcinia gardneriana (Planch. \& Triana) Zappi & $\mathrm{x}$ & $\mathrm{x}$ & & St & FI & 5 & 1,0000 \\
\hline Rheedia gardneriana Planch. \& Triana & & $\mathrm{x}$ & & $\mathrm{St}$ & FI & 10 & 1,0000 \\
\hline Tovomitopsis saldanhae Engler & $\mathrm{x}$ & & & St & FM & 10 & 1,0000 \\
\hline \multicolumn{8}{|l|}{ ELAEOCARPACEAE } \\
\hline Sloanea sp. & & $\mathrm{x}$ & & $\mathrm{Sc}$ & FI & 10 & 1,0000 \\
\hline \multicolumn{8}{|l|}{ ERYTHROXYLACEAE } \\
\hline Erythroxylum deciduum A. St.-Hil. & & $\mathrm{x}$ & & $\mathrm{P}$ & FI & 50 & 0,0053 \\
\hline \multicolumn{7}{|l|}{ EUPHORBIACEAE } & 0,0008 \\
\hline Acalypha sp. & $\mathrm{x}$ & & & Sc & FM & 20 & 0,3087 \\
\hline Croton sp. & $\mathrm{x}$ & $\mathrm{x}$ & & Sc & FI & 55 & 0,0039 \\
\hline
\end{tabular}




\begin{tabular}{|c|c|c|c|c|c|c|c|}
\hline \multicolumn{8}{|l|}{$\begin{array}{l}\text { Sapium glandulatum (Vell.) Pax } \\
\text { FABACEAE }\end{array}$} \\
\hline Acacia polyphylla DC. & & $\mathrm{x}$ & & $\mathrm{P}$ & FI & 10 & 1,0000 \\
\hline Anadenanthera macrocarpa (Benth.) Brenan & $\mathrm{x}$ & $\mathrm{x}$ & & $\mathrm{Si}$ & FI & 47,8 & 0,0281 \\
\hline Anadenanthera peregrina (L.) Speg. & & & $\mathrm{x}$ & $\mathrm{P}$ & PAS & 10 & 1,0000 \\
\hline Dalbergia nigra (Vell.) Allemao ex Benth. & & $\mathrm{x}$ & & $\mathrm{Si}$ & FI & 60 & 0,0013 \\
\hline Copaifera langsdorffii Desf. & $\mathrm{x}$ & & & St & FM & 10 & 1,0000 \\
\hline Inga edulis Mart. & $\mathrm{x}$ & & & $\mathrm{Si}$ & FM & 50 & 0,0050 \\
\hline Inga marginata Willd. & $\mathrm{x}$ & & & $\mathrm{Si}$ & FM & 30 & 0,0923 \\
\hline Machaerium brasiliense Vogel & & $\mathrm{x}$ & & $\mathrm{Si}$ & FI & 20 & 0,3081 \\
\hline Machaerium nyctitans (Vell.) Benth. & $\mathrm{x}$ & & & $\mathrm{Si}$ & FM & 10 & 1,0000 \\
\hline Machaerium stiptatum (DC.) Vogel & $\mathrm{x}$ & & & $\mathrm{Si}$ & FM & 10 & 1,0000 \\
\hline Peltophorum dubium (Spreng.) Taub. & & $\mathrm{x}$ & $\mathrm{x}$ & $\mathrm{P}$ & FI & 13,3 & 0,7566 \\
\hline Piptadenia gonoacantha (Mart.) J.F. Macbr. & $\mathrm{x}$ & $\mathrm{x}$ & $\mathrm{x}$ & $\mathrm{Si}$ & FI & 10 & 1,0000 \\
\hline Schizolobium parahyba (Vell.) S.F. Blake & & & $\mathrm{x}$ & $\mathrm{P}$ & PAS & 10 & 1,0000 \\
\hline Senna multijuga (Rich.) H.S. Irwin \& Barneby & & $\mathrm{x}$ & $\mathrm{x}$ & $\mathrm{Si}$ & FI & 5 & 1,0000 \\
\hline Senna obtusifolia (L.) H.S. Irwin \& Barneby & & & $\mathrm{x}$ & $\mathrm{Sc}$ & PAS & 20 & 0,3089 \\
\hline Stryphnodendron polyphyllum Mart. & & $\mathrm{x}$ & & $\mathrm{P}$ & FI & 10 & 1,0000 \\
\hline Swartzia myrtifolia $\mathrm{Sm}$. & $\mathrm{x}$ & & & St & FM & 20 & 0,3105 \\
\hline \multicolumn{8}{|l|}{ HYPERICACEAE } \\
\hline Vismia guianensis (Aubl.) Pers. & & $\mathrm{x}$ & & $\mathrm{P}$ & FI & 30 & 0,0870 \\
\hline \multicolumn{8}{|l|}{ LAURACEAE } \\
\hline Nectandra lanceolata (Ruiz \& Pav.) Mez. & $\mathrm{x}$ & $\mathrm{x}$ & & St & FM & 27 & 0,0833 \\
\hline Nectandra oppositifolia Nees \& Mart. & & $\mathrm{x}$ & & St & FI & 80 & $0.0001 *$ \\
\hline Nectandra reticulata Mez. & $\mathrm{x}$ & $\mathrm{x}$ & & St & FM & 77,6 & $0.0001 *$ \\
\hline Nectandra saligna Nees & $\mathrm{x}$ & $\mathrm{x}$ & & St & FM & 76 & $0.0002 *$ \\
\hline Nectandra sp. & $\mathrm{x}$ & & & $\mathrm{Sc}$ & FM & 20 & 0,3110 \\
\hline Ocotea odorífera Rohwer & $\mathrm{x}$ & & & St & FM & 10 & 1,0000 \\
\hline \multicolumn{8}{|l|}{ LECYTHIDACEAE } \\
\hline Eschweilera sp. & $\mathrm{x}$ & & & $\mathrm{Sc}$ & FM & 20 & 0,3119 \\
\hline \multicolumn{8}{|l|}{ MALVACEAE } \\
\hline Luehea grandiflora Mart. & & $\mathrm{x}$ & & $\mathrm{Si}$ & FI & 30 & 0,0947 \\
\hline Pseudobombax grandiflorum (Cav.) A. Robyns & & $\mathrm{x}$ & & $\mathrm{Si}$ & FI & 10 & 1,0000 \\
\hline Triumfetta bartramii $\mathrm{L}$. & & & $\mathrm{x}$ & $\mathrm{Sc}$ & PAS & 10 & 1,0000 \\
\hline \multicolumn{8}{|l|}{ MELASTOMATACEAE } \\
\hline Leandra niangaeformis Cogn. & & $\mathrm{x}$ & & Sc & FI & 60 & 0,0012 \\
\hline Miconia hymenonervia (Raddi) Cogn. & $\mathrm{x}$ & $\mathrm{x}$ & & $\mathrm{Si}$ & FM & 6,7 & 1,0000 \\
\hline Miconia pusilliflora Beurl. & $\mathrm{x}$ & & & $\mathrm{P}$ & FM & 50 & 0,0050 \\
\hline \multicolumn{8}{|l|}{ MELIACEAE } \\
\hline Cabralea canjerana (Vell.) Mart. & $\mathrm{x}$ & & & $\mathrm{Si}$ & FM & 50 & 0,0051 \\
\hline Guarea macrophylla Vahl & $\mathrm{x}$ & $\mathrm{x}$ & & St & FM & 90,6 & $0.0001 *$ \\
\hline Trichilia catigua A. Juss. & $\mathrm{x}$ & & & St & FM & 10 & 1,0000 \\
\hline Trichilia lepidota Mart. & $\mathrm{x}$ & $\mathrm{x}$ & & St & FI & 52,3 & 0,0220 \\
\hline Trichilia pallida $\mathrm{Sw}$. & $\mathrm{x}$ & & & St & FM & 10 & 1,0000 \\
\hline Trichilia sp. & $\mathrm{x}$ & & & $\mathrm{Sc}$ & FM & 10 & 1,0000 \\
\hline \multicolumn{8}{|l|}{ MONIMIACEAE } \\
\hline Mollinedia widgrenii A. DC. & $\mathrm{x}$ & & & St & FM & 90 & $0.0001 *$ \\
\hline \multicolumn{8}{|l|}{ MORACEAE } \\
\hline Brosimum guianense (Aubl.) Huber & & $\mathrm{x}$ & & $\mathrm{Si}$ & FI & 10 & 1,0000 \\
\hline $\begin{array}{l}\text { Sorocea bonplandii (Baill.) W.C. Burger, Lanj. \&Wess. } \\
\text { MYRISTICACEAE }\end{array}$ & $\mathrm{x}$ & $\mathrm{x}$ & & St & FM & 91,2 & $0.0001 *$ \\
\hline Virola oleifera (Schott) A.C. Smith & $\mathrm{x}$ & $\mathrm{x}$ & & $\mathrm{Si}$ & FM & 25,7 & 0,2192 \\
\hline
\end{tabular}




\begin{tabular}{|c|c|c|c|c|c|c|c|}
\hline \multicolumn{8}{|l|}{ MYRTACEAE } \\
\hline Campomanesia xanthocarpa O. Berg & $\mathrm{x}$ & & & St & FM & 10 & 1,0000 \\
\hline Eugenia florida DC. & & $\mathrm{x}$ & & $\mathrm{P}$ & FI & 10 & 1,0000 \\
\hline Eugenia sp. & $\mathrm{x}$ & $\mathrm{x}$ & & $\mathrm{Sc}$ & FI & 5 & 1,0000 \\
\hline Myrcia brasiliensis Kiaersk. & $\mathrm{x}$ & & & St & FM & 80 & $0.0001 *$ \\
\hline Myrcia fallax (Rich.) DC. & $\mathrm{x}$ & $\mathrm{x}$ & & $\mathrm{Si}$ & FM & 30 & 0,1553 \\
\hline Myrcia rostrata DC. & & $\mathrm{x}$ & & $\mathrm{P}$ & FI & 20 & 0,3097 \\
\hline Myrcia sp. & & $\mathrm{x}$ & & $\mathrm{Sc}$ & FI & 10 & 1,0000 \\
\hline Myrtaceae sp.1 & $\mathrm{x}$ & $\mathrm{x}$ & & $\mathrm{Sc}$ & FM & 16 & 0,3130 \\
\hline Myrtaceae sp.2 & & $\mathrm{x}$ & & $\mathrm{Sc}$ & FI & 10 & 1,0000 \\
\hline Plinia sp. & $\mathrm{x}$ & & & $\mathrm{Sc}$ & FM & 10 & 1,0000 \\
\hline $\begin{array}{l}\text { Psidium guajava } \mathrm{L} . \\
\text { NYCTAGINACEAE }\end{array}$ & & & $\mathrm{x}$ & $\mathrm{P}$ & PAS & 10 & 1,0000 \\
\hline $\begin{array}{l}\text { NYCTAGINACEAE } \\
\text { Guapira opposita (Vell.) Reitz }\end{array}$ & \multicolumn{7}{|c|}{ NYCTAGINACEAE } \\
\hline \multicolumn{8}{|l|}{ PICRAMNIACEAE } \\
\hline \multicolumn{8}{|l|}{ PIPERACEAE } \\
\hline Ottonia sp. & $\mathrm{x}$ & & & $\mathrm{Sc}$ & FM & 20 & 0,3172 \\
\hline Pothomorphe umbellata (L.) Miq. & & $\mathrm{x}$ & & $\mathrm{Si}$ & FI & 10 & 1,0000 \\
\hline Piper aduncum L. & & $\mathrm{x}$ & & $\mathrm{P}$ & FI & 30 & 0,0857 \\
\hline Piper arboreum Aubl. & $\mathrm{x}$ & $\mathrm{x}$ & & $\mathrm{P}$ & FI & 37,1 & 0,0406 \\
\hline \multirow{2}{*}{\multicolumn{8}{|c|}{ ROSACEAE }} \\
\hline & & & & & & & \\
\hline Prunus sellowii Koehne & $\mathrm{x}$ & & & $\mathrm{Si}$ & FM & 10 & 1,0000 \\
\hline \multicolumn{8}{|l|}{ RUBIACEAE } \\
\hline Amaioua guianensis Aubl. & $\mathrm{x}$ & & & St & FM & 30 & 0,0907 \\
\hline Coffea arabica $\mathrm{L}$. & $\mathrm{x}$ & $\mathrm{x}$ & & $\mathrm{Sc}$ & FM & 78,6 & $0.0003^{*}$ \\
\hline Psychotria carthagenensis Jacq. & $\mathrm{x}$ & & & St & $\mathrm{FM}$ & 60 & 0,0006 \\
\hline Psychotria hastisepala Müll. Arg. & $\mathrm{x}$ & $\mathrm{x}$ & & St & FM & 72 & $0.0002 *$ \\
\hline Psychotria longepedenculata Gardiner & $\mathrm{x}$ & $\mathrm{x}$ & & St & FM & 68,2 & 0,0004 \\
\hline Psychotria myriantha Müll. Arg. & $\mathrm{x}$ & $\mathrm{x}$ & & St & FM & 40 & 0,0246 \\
\hline Psychotria nиda (Cham. \& Schltdl.) Wawra & $\mathrm{x}$ & $\mathrm{x}$ & & St & FM & 35 & 0,0602 \\
\hline Psychotria sessilis Vell. & $\mathrm{x}$ & $\mathrm{x}$ & & $\mathrm{Si}$ & FI & 99,9 & $0.0001 *$ \\
\hline Psychotria sp.1 & $\mathrm{x}$ & & & $\mathrm{Sc}$ & FM & 10 & 1,0000 \\
\hline Psychotria sp.2 & $\mathrm{x}$ & & & $\mathrm{Sc}$ & FM & 30 & 0,0899 \\
\hline Randia armata (Sw.) DC. & $\mathrm{x}$ & & & St & $\mathrm{FM}$ & 10 & 1,0000 \\
\hline \multicolumn{8}{|l|}{ RUTACEAE } \\
\hline Citrus limonia (L.) Osbeck & $\mathrm{x}$ & & & Sc & FM & 10 & 1,0000 \\
\hline Dyctioloma vandellianum DC. & & $\mathrm{x}$ & & $\mathrm{Si}$ & FI & 10 & 1,0000 \\
\hline \multicolumn{8}{|l|}{ SALICACEAE } \\
\hline Casearia decandra Jacq. & & $\mathrm{x}$ & & St & FI & 10 & 1,0000 \\
\hline Casearia gossypiosperma Briq. & $\mathrm{x}$ & $\mathrm{x}$ & & $\mathrm{Si}$ & FI & 13,3 & 0,7481 \\
\hline \multicolumn{8}{|l|}{ SAPINDACEAE } \\
\hline Allophylus edulis (A. St.-Hill, Cambess. \& Juss.) Radlk. & $\mathrm{x}$ & $\mathrm{x}$ & & $\mathrm{P}$ & FM & 7,5 & 1,0000 \\
\hline Allophylus sericeus Radlk. & $\mathrm{x}$ & & & $\mathrm{P}$ & FM & 10 & 1,0000 \\
\hline Matayba elaeagnoides Radlk. & $\mathrm{x}$ & $\mathrm{x}$ & & $\mathrm{Si}$ & FI & 21,4 & 0,1863 \\
\hline \multicolumn{8}{|l|}{ POTACEAE } \\
\hline $\begin{array}{l}\text { Chrysophyllum flexuosum Mart. } \\
\text { SIPARUNACEAE }\end{array}$ & $\mathrm{x}$ & & & St & $\mathrm{FM}$ & 10 & 1,0000 \\
\hline \multicolumn{5}{|l|}{ SIPARUNACEAE } & & & \\
\hline Siparuna reginae (Tul.) A. DC. & $\mathrm{x}$ & & & $\mathrm{Sc}$ & $\mathrm{FM}$ & 20 & 0,3172 \\
\hline \multicolumn{8}{|l|}{ SOLANACEAE } \\
\hline Cestrum sp. & $\mathrm{x}$ & & & $\mathrm{Sc}$ & FM & 20 & 0,3127 \\
\hline Solanum cernuum Vell. & & & $\mathrm{x}$ & $\mathrm{P}$ & PAS & 10 & 1,0000 \\
\hline
\end{tabular}




\begin{tabular}{|c|c|c|c|c|c|c|}
\hline Solanum erianthum D. Don & & $\mathrm{x}$ & $\mathrm{P}$ & PAS & 50 & 0,0049 \\
\hline Solanum leucodendron Sendtn. & $\mathrm{x}$ & & $\mathrm{Sc}$ & FI & 10 & 1,0000 \\
\hline $\begin{array}{l}\text { Solanum lycocarpum A. St.-Hil. } \\
\text { VERBENACEAE }\end{array}$ & & $\mathrm{x}$ & $\mathrm{P}$ & PAS & 10 & 1,0000 \\
\hline Lantana camara $\mathrm{L}$. & & $\mathrm{x}$ & $\mathrm{P}$ & PAS & 20 & 0,3103 \\
\hline
\end{tabular}

FEA: floresta em estágio avançado de regeneração; FEM: floresta em estágio médio de regeneração; PAS: pastagem; GS: grupo sucessional; GI: grupo indicador; IV: valor indicador (\%); p: grau de significância; P: pioneira; Si: secundária inicial; St: secundária tardia; SC: sem caracterização; *espécies indicadoras que tiveram no mínimo 70\% de IV e p: 0,05.

Seguindo o mesmo padrão de $P$. sessilis no trecho de floresta em estágio médio, Vernonia polyanthes também apresentou alta densidade e dominância no trecho de pastagem abandonada, se destacando em VI na comunidade (Tabela 2). Em nascentes dominadas por pastagem (Brachiaria sp.), mas em processo de regeneração natural, essa espécie foi uma das maiores em VI (FERREIRA et al., 2009) e também foi registrada em pequenos fragmentos (5-14 ha) de floresta ombrófila no sudeste do Brasil (TABARELLI et al., 1999). Essa espécie é caracterizada como pioneira (FERREIRA et al., 2009), o que evidencia sua importância para a recuperação de áreas degradadas ou perturbadas, dando início ao processo de sucessão ecológica.

$\mathrm{Na}$ análise de DCA, utilizando a abundância como parâmetro estrutural, cada trecho formou um grupo próprio (Figura 1), evidenciando claramente a distinção estrutural entre eles. No Eixo I, as espécies que apresentaram os escores mais baixos ocorrem exclusivamente no trecho de pastagem, caracterizadas como pioneiras, e aquelas de escore mais alto foram as com maior abundância na floresta em estágio médio de regeneração, pertencentes ao grupo das secundárias iniciais e secundárias tardias. O Eixo II é dominado pelas espécies Piper arboreum Aubl., Anadenanthera macrocarpa e Xylopia sericea, que foram amostradas em pelo menos dois trechos da EPTEA Mata do Paraíso. Essas espécies são características de áreas em processo inicial a intermediário de sucessão, evidenciando o estágio sucessional em que o trecho de floresta em estágio médio se encontra, onde foram mais representativas em termos de abundância. Já as espécies com escores baixos no Eixo II, Machaerium braziliensis Vogel, Erythroxyllum pelleterianum e Matayba elaeagnoides Radlk., foram amostradas apenas nos trechos de floresta, mas a abundância de cada uma é maior na FEM do que na FEA, possivelmente por serem espécies caracterizadas como secundárias iniciais.

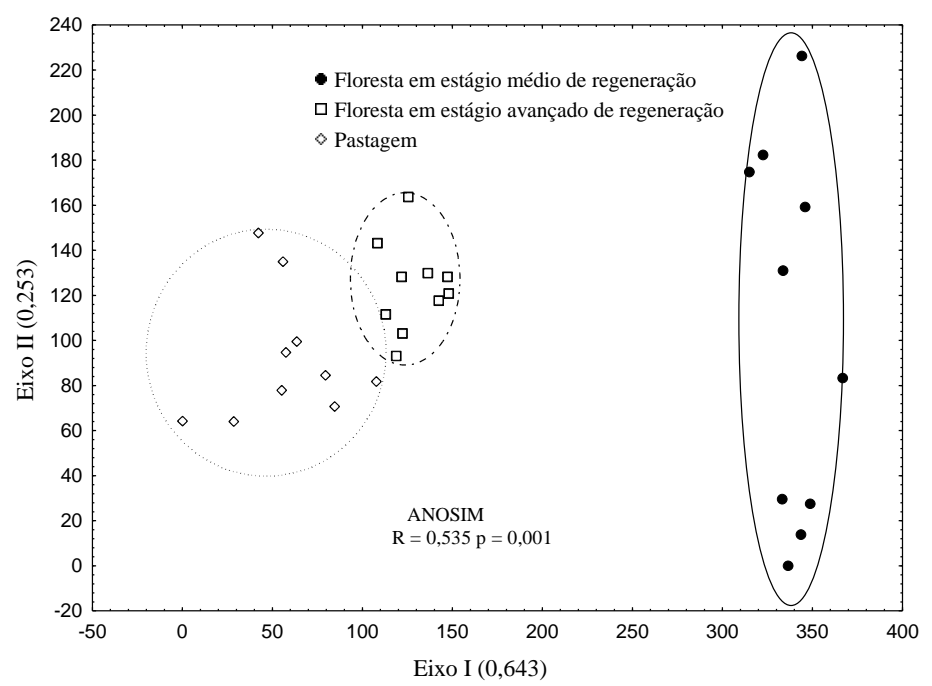

Figura 1. Resultados gráficos dos dois primeiros eixos da Análise de Correspondência Retificada (DCA) e Análise de Similaridade Florística Bifatorial (ANOSIM) para as diferentes áreas amostradas em Viçosa, MG.

Figure 1. Graphical results of the two first axes of Detrended Correspondence Analysis (DCA) and Floristic Bifactorial Analysis of Similarity (ANOSIM) for the different sampled areas in Viçosa, MG. 
Chazdon et al. (2007) destacam que é importante considerar se a composição florística da regeneração natural em florestas tropicais secundárias está convergindo às florestas maduras do entorno ou se permanecem com composições florísticas distintas. Aliada à dissimilaridade estrutural, a flora da comunidade da regeneração natural é composta por diferentes espécies entre os trechos, pois a Análise de Similaridade Bifatorial indicou que há diferença significativa entre os mesmos (ANOSIM $r=0,535$; $\mathrm{p}=0,001)$.

Essa variação estrutural e florística entre os trechos sugere que a matriz florestal é formada por diferentes mosaicos sucessionais que ainda mantêm suas próprias características, não havendo direcionamento para a conversão a um único estágio sucessional (floresta madura). As variações encontradas em termos de riqueza, diversidade e composição florística, assim como na abundância das espécies entre os trechos, principalmente a pastagem, demonstram que a matriz florestal não segue o padrão comum relatado por Chazdon et al. (2009), em que áreas com perturbações antrópicas situadas próximas a fragmentos florestais podem exibir, ao longo do tempo, elevada riqueza florística.

De acordo com a análise de espécies indicadoras, 34 espécies (30\%) apresentaram distribuição significativamente diferenciada $(\mathrm{p}<0,05)$, mas considerando o valor indicador adotado $(70 \%)$ são apenas 15 espécies (Tabela 3). As espécies Coffea arabica, Euterpe edulis, Guapira opposita, Guarea macrophylla, Mollinedia widgrenii A. DC., Myrcia brasiliensis Kiaersk., Nectandra reticulata, Nectandra saligna Nees, Picramnia regnelli Engl., Psychotria hastisepala e Sorocea bonplandii foram indicadoras da área de floresta em estágio avançado de regeneração; Nectandra oppositifolia, Psychotria sessilis e Siparuna guianensis, da área de floresta em estágio médio; e Vernonia polyanthes, da pastagem, cujo resultado mostra que tais espécies possuem distribuição restrita a cada estágio sucessional, não sendo comumente encontradas entre as áreas. Certamente, os estágios sucessionais em que se encontra a matriz florestal possibilitam grande variedade de habitats e, por conseguinte, o estabelecimento de espécies preferenciais nesses habitats.

Considerando as 34 espécies que apresentaram distribuição significativamente diferenciada, aquelas indicadoras do trecho de floresta em estágio médio estão uniformemente distribuídas entre os grupos sucessionais, enquanto a maioria daquelas de floresta em estágio avançado de regeneração são classificadas como secundárias tardias, e da pastagem, como pioneiras.

\section{CONCLUSÕES}

- O componente da regeneração natural do trecho de floresta em estágio médio e de pastagem ainda mantém suas características próprias, com maior representatividade de espécies secundárias iniciais na FEM e pioneiras na PAS.

- Os resultados de estrutura e composição florística reforçam as particularidades de cada trecho, permanecendo assim a classificação segundo a resolução do CONAMA quanto à vegetação secundária de regeneração.

- Embora a sucessão secundária não seja estática e direcional, a integridade da matriz florestal poderia ser suficiente para conduzir a regeneração natural de tais áreas para um ambiente com maior diversidade florística e com espécies que poderiam indicar um avanço na sucessão.

\section{AGRADECIMENTOS}

Ao CNPq, pela bolsa de doutorado à primeira autora (Programa de Pós-Graduação em Ciência Florestal/UFV), e à administração da EPTEA Mata do Paraíso, pela oportunidade para o desenvolvimento do estudo.

\section{REFERÊNCIAS}

AIDE, T. M.; ZIMMERMAN, J. K.; PASCARELLA, J. B.; RIVERA, L.; MARCANO-VERGA, H. Forest regeneration in a chronosequence of tropical abandoned pastures: implications for restoration ecology. Restoration Ecology, Washington, v. 8, p. 328 - 338, 2000.

ANDEL, T. V. Floristic composition and diversity of mixed primary and secondary forests in northwest Guyana. Biodiversity and Conservation, Berlin, v. 10, p. 1645 - 1682, 2001. 
Angiosperm Philogeny Group (APG) III. An update of the Angiosperm Phylogeny Group classification for the orders and families of flowering plants: APG III. Botanical Journal of the Linnean Society, London, v. 161, p. 105 - 121, 2009.

ARAÚJO, F. S. de; MARTINS, S. V.; MEIRA NETO, J. A. A.; LANI, J. L.; PIRES, I. E. Estrutura da vegetação arbustivo-arbórea colonizadora de uma área degradada por mineração de Caulim, Brás Pires, MG. Revista Árvore, Viçosa, v. 30, p. 107 - 116, 2006.

ARMELIN, R. S.; MANTOVANI, W. Definições de clareira natural e suas implicações no estudo da dinâmica sucessional em florestas. Rodriguésia, Rio de Janeiro, v. 52, p. 5 - 15, 2001.

BRASIL. Resolução CONAMA n. 392, de 25 de junho de 2007. Define vegetação primária e secundária de regeneração de Mata Atlântica no estado de Minas Gerais. Diário Oficial da União, Brasília, DF, 26 jun. 2007. Disponível em: <http://www.mma.gov.br/estruturas/202/_arquivos/conama_res_cons_2007_ 392_estgios_sucessionais_de_florestas_mg_202.pdf>. Acesso em: 16/01/2013.

BRAZ, D. M.; CARVALHO-OKANO, R. M.; KAGEYAMA, C. Acanthaceae da Reserva Florestal Mata do Paraíso, Viçosa, Minas Gerais. Revista Brasileira de Botânica, São Paulo, v. 25, p. 495 - 504, 2002.

CASTRO, P. S.; VALENTE, O. F.; COELHO, D. T.; RAMALHO, R. S. Interceptação da chuva por mata natural secundária na região de Viçosa, MG. Revista Árvore, Viçosa, v. 7, p. 76 - 89, 1983.

CAVELIER, J.; AIDE, T. M.; SANTOS, C.; EUSSE, A. M.; DUPUY, J. M. The savannization of mois forests in the Sierra Nevada de Santa Marta, Colombia. Journal of Biogeography, New York, v. 25, p. $901-912,1998$.

CHAZDON, R. L.; LETCHER, S. G.; VAN BREUGEL, M.; MARTÍNEZ-RAMOS, M.; BONGERS, F.; FINEGAN, B. Rates of change in tree communities of secondary neotropical forests following major disturbances. Philosophical Transactions of the Royal Society B, London, v. 362, p. 273 - 289, 2007.

CHAZDON, R. L.; PERES, C. A.; DENT, D.; SHEIL, D.; LUGO, A. E.; LAMB, D.; STORK, N. E.; MILLER, S. E. The potential for species conservation in tropical secondary forests. Conservation Biology, New York, v. 23, n. 6, p. 1406 - 1417, 2009.

CONNELL, J. H.; SLATYER, R. O. Mechanisms of succession in natural communities and their role in community stability and organization. American Naturalist, Chicago, v. 111, p. 1119 - 1144, 1977.

CORREAA, G. F. Modelo de evolução e mineralogia da fração argila de solos do planalto de Viçosa, MG. 86 f. Dissertação (Mestrado em Solos e Nutrição de Plantas) - Universidade Federal de Viçosa, Viçosa, 1984.

DIAS, A. S.; FERREIRA JÚNIOR, W. G.; SILVA, A. F.; CARMO, F. M. S. Dinâmica da regeneração natural e a presença de Coffea arabica influenciando a diversidade de espécies. In: CONGRESSO DE ECOLOGIA DO BRASIL, 7., 2005, Caxambu. Anais... Caxambu, 2005.p. 1 - 2.

DUFRÊNE, M.; LEGENDRE, P. Species assemblages and indicator species: the need for a flexible asymmetrical approach. Ecological Monographs, New York, v. 67, p. 345 - 366, 1997.

DURIGAN, G.; NISHIKAWA, D. L. L.; ROCHA, E.; SILVEIRA, E. R.; PULITANO, F. M.; REGALADO, L. B.; CARVALHAES, M. A.; PARANAGUÁ, P. A.; RANIERI, V. E. L. Caracterização de dois estratos da vegetação em uma área de cerrado no município de Brotas, SP, Brasil. Acta Botanica Brasilica, São Paulo, v. 16, p. 251 - 262, 2002.

FERREIRA, M. J.; PEREIRA, I. M.; BOTELHO, S. A.; MELLO, C. R. Avaliação da regeneração natural em pastagens perturbadas no município de Lavras, MG. Ciência Florestal, Santa Maria, v. 19, p. 109 129, 2009.

GANDOLFI, S.; LEITÃO-FILHO. H. de F.; BEZERRA, C. L. Levantamento florístico e caráter sucessional das espécies arbustivo-arbóreas de uma floresta mesófila semidecídua no município de Guarulhos, SP. Revista Brasileira de Biologia, São Paulo, v. 55, p. 753 - 767, 1995. 
GASPARINO, D.; MALAVASI, U. C.; MALAVASI, M. M.; SOUZA, I. de. Quantificação do banco de sementes sob diferentes usos do solo em área de domínio ciliar. Revista Árvore, Viçosa, v. 30, p. 1 - 9 , 2006.

GOMIDE, L. R.; SCOLFORO, J. R. S.; OLIVEIRA, A. D. Análise da diversidade e similaridade de fragmentos florestais nativos na bacia do rio São Francisco, em Minas Gerais. Ciência Florestal, Santa Maria, v. 16, p. 127 - 144, 2006.

HAMMER, O.; HARPER, D. A. T.; RIAN, P. D. Past: palaeonthological statistics software package for education and data analysis. Version. 1.37. Disponível em: <http://palaeoelectronica.org/2001_1/past/issue1_01.htm>. Acesso em: 11/10/2012.

HIGUCHI, P.; REIS, M. G. F.; REIS, G. G.; PINHEIRO, A. L.; SILVA, C. T.; OLIVEIRA, C. H. R. Composição florística da regeneração natural de espécies arbóreas ao longo de oito anos em um fragmento de Floresta Estacional Semidecidual, em Viçosa, MG. Revista Árvore, Viçosa, v. 30, p. 893 904, 2006.

HILL, M. O.; GAUCH, H. G. Detrended Correspondence Analysis, an improved ordination technique. Vegetatio, Berlin, v. 42, p. 47 - 58, 1980.

HOOPER, E.; LEGENDRE, P.; CONDIT, R. Barriers to forest regeneration of deforested and abandoned land in Panama. Journal of Applied Ecology, London, v. 42, p. 1165 - 1174, 2005.

HUTCHESON, K. A test for comparing diversities based on the Shannon formula. Journal Theory Biology, Amsterdam, v. 29, p. 151 - 154, 1970.

INSTITUTO BRASILEIRO DE GEOGRAFIA E ESTATÍSTICA (IBGE). Manual técnico da vegetação brasileira: sistema fitogeográfico, inventário das formações florestais e campestres, técnicas e manejo de coleções botânicas, procedimentos para mapeamentos. Rio de Janeiro: IBGE, 2012. 2 ed. 271 p.

KÖPPEN, W. P. Climatologia: con un estudio de los climas de la tierra. México: Fondo de Cultura Economica, 1948. 478 p.

KUNZ, S. H. O banco de sementes do solo e a regeneração natural em diferentes estádios sucessionais de Floresta Estacional Semidecidual e de pastagem abandonada, Reserva Mata do Paraíso, Viçosa, MG. 84 p. Tese (Doutorado em Ciência Florestal) - Universidade Federal de Viçosa, Viçosa, 2011.

MAGURRAN, A. E. Medindo a diversidade biológica. Curitiba: UFPR, 2013. 261 p.

MARTINEZ-GARZA, C.; TOBÓN, W.; CAMPO, J.; HOWE, H. F. Drought mortality of tree seedlings in an eroded tropical pasture. Land Degradation and Development, New York, v. 22, 2011.

MARTINS, S. V.; COLLETI JÚNIOR, R.; RODRIGUES, R. R.; GANDOLFI, S. Colonization of gaps produced by death of bamboo clumps in a semideciduous mesophytic forest in south-eastern Brazil. Plant Ecology, Berlin, v. 172, p. 121 - 131, 2004.

MARTINS, S. V.; GLERIANI, J. M.; AMARAL, C. H.; RIBEIRO, T. M. Caracterização do dossel e do estrato de regeneração natural no sub-bosque e em clareiras de uma Floresta Estacional Semidecidual no município de Viçosa, MG. Revista Árvore, Viçosa, v. 32, p. 759 - 767, 2008.

MARTINS, S. V.; RIBEIRO, G. A. Initial secondary succession in a forest fragment disturbed by fire in Viçosa, MG. In: VIEGAS, D. X. Forest fire research \& wildland fire safety. Rotterdam, 2002. p. 213.

MARTINS, S. V.; RODRIGUES, R. R. Gap-phase regeneration in a semideciduous mesophytic forest, south-eastern Brazil. Plant Ecology, Berlin, v. 163, p. 51 - 62, 2002.

McCUNE, B.; MEFFORD, M. J. Multivariate analysis of ecological data. Oregon: Gleneden Beach, 2006. $237 \mathrm{p}$.

MISSOURI BOTANICAL GARDEN. Tropicos. Disponível em: 〈http://www.tropicos.org/Home.aspx>. Acesso em: 10/11/2012. 
MORELLATO, L. P. C.; HADDAD, C. F. B. Introduction: the brazilian atlantic forest. Biotropica, New York, v. 32, p. 786 - 792, 2000.

MUELLER-DOMBOIS, D.; ELLEMBERG, H. Aims and methods vegetation ecology. New York: Wiley, 1974. $547 \mathrm{p}$.

NOTMAN, E.; GORCHOV, D. L. Variation in post-dispersal seed predation in mature peruvian lowland tropical forest and fallow agricultural sites. Biotropica, New York, v. 33, p. 621 - 636, 2001.

PEREIRA, I. M.; ANDRADE, L. A. de; COSTA, J. R. M.; DIAS, J. M. Regeneração natural em um remanescente de caatinga sob diferentes níveis de perturbação, no agreste paraibano. Acta Botanica Brasilica, São Paulo, v. 15, p. 421 - 426, 2001.

PINTO, S. I. C.; MARTINS, S. V.; SILVA, A. G.; BARROS, N. F.; DIAS, H. C. T.; SCOSS, L. M. Estrutura do componente arbustivo-arbóreo de dois estágios sucessionais de Floresta Estacional Semidecidual na Reserva Florestal Mata do Paraíso, Viçosa, MG, Brasil. Revista Árvore, Viçosa, v. 31, p. 823 - 833, 2007.

R DEVELOPMENT CORE TEAM. R: a language and environment for statistical computing. Austria: R Foundation for Statistical Computing, 2009.

RAYOL, B. P.; ALVINO, F. de O.; SILVA, M. F. F. da. Estrutura e composição florística da regeneração natural de duas florestas secundárias em Capitão Poço, Pará, Brasil. Amazônia: Ciência \& Desenvolvimento, Manaus, v. 4, p. 103 - 116, 2008.

RIBAS, R. F.; MEIRA-NETO, J. A. A.; SILVA, A. F.; SOUZA, A. L. Composição florística de dois trechos em diferentes etapas serais de uma Floresta Estacional Semidecidual em Viçosa, Minas Gerais. Revista Árvore, Viçosa, v. 27, p. 821 - 830, 2003.

SALDARRIAGA, J. G.; WEST, D. C.; THARP, M. L.; UHL, C. Long-term chronosequence of forest succession in the upper Rio Negro of Colombia and Venezuela. Journal of Ecology, London, v. 76, p. 938 - 958, 1988.

SANTOS, H. G. dos; JACOMINE, P. K. T.; ANJOS, L. H. C. dos; OLIVEIRA, V. A. de; LUMBRERAS, J. F.; COELHO, M. R.; AlMEIDA, J. A.; CUNHA, T. J. F.; OlIVEIRA, J. B. de. (Eds.). Sistema brasileiro de classificação de solos. 3. ed. Brasília: Embrapa, 2013. 353 p.

SCHORN, L. A.; GALVÃO, F. Dinâmica da regeneração natural em três estágios sucessionais de uma Floresta Ombrófila Densa em Blumenau, SC. Revista Floresta, Curitiba, v. 36, p. 59 - 74, 2006.

SHEPHERD, G. J. Fitopac 2.01 - manual do usuário. Campinas: UNICAMP, 2009. 64 p.

SILVA JÚNIOR, W. M.; MARTINS, S. V.; SILVA, A. F.; DE MARCO, P. Regeneração natural de espécies arbustivo-arbóreas em dois trechos de uma Floresta Estacional Semidecidual, Viçosa, MG. Scientia Forestalis, Piracicaba, v. 66, p. 69 - 79, 2004.

SOUZA, P. B.; MARTINS, S. V.; COSTALONGA, S. R.; COSTA, G. O. Florística e estrutura da vegetação arbustivo-arbórea do sub-bosque de um povoamento de Eucalyptus grandis W. Hill ex Maiden em Viçosa, MG, Brasil. Revista Árvore, Viçosa, v. 31, p. 533 - 543, 2007.

TABARELLI, M.; MANTOVANI, W.; PERES, C. A. Effects of habitat fragmentation on plant guil structure in the montane Atlantic Forest of southeastern Brazil. Biological Conservation, Amsterdam, v. 91 , p. $119-127,1999$. 

Kunz, S. H.; Martins, S. V. 